\title{
CONCEITOS PARA REDUÇÃO DE SUBPRODUTOS SEM VALOR AGREGADO NA CONSTRUÇÃO CIVIL: ECONOMIA CIRCULAR, DESIGN FOR DECONSTRUCTION E PRÉ-FABRICADOS
}

\author{
Drielly Pena Cesar (UNIARA) driellypenna@gmail.com \\ José Camilo Barbosa (UNIARA) camilobarbosa@ hotmail.com \\ José Luís Garcia Hermosilla (UNIARA) jlghermosilla@ hotmail.com
}

\section{Resumo}

O setor da construção civil apresenta impactos significativos sobre o meio ambiente por gerar subprodutos sem valor agregado durante o ciclo vida, encontrados na construção e demolição (CD). Por isso, a adoção de conceitos e práticas como a Economia Circular (EC), uso de préfabricados assim como o Design for Deconstruction (DfD), são apresentados como métodos para reduzir esses subprodutos. Objetivo dessa pesquisa foi realizar uma revisão bibliográfica de forma exploratória na base de dados Science Direct, com a combinação das palavras-chaves e o uso do software Vosviewer, a fim de estabelecer a relação entre elas sobre a geração de excedentes sem valor agregado, denominados em muitos estudos de resíduos ou desperdícios. Conclui-se com o estudo que o conceito de economia circular apresenta maior ascensão nos últimos anos, contudo a integração dos três conceitos não está consolidada nas pesquisas.

Palavras-Chaves: Economia circular; Resíduos de construção e demolição; Design for deconstruction; Pré-fabricados; Desperdício.

\section{Introdução}

A indústria da construção civil é um dos setores da economia que exerce grande impacto sobre o meio ambiente, devido ao uso excessivo de recursos naturais. Além disso, consolida-se como uma grande geradora de resíduos durante todo o ciclo de vida, incorporando as atividades de construção e demolição (CD) (UNITED NATIONS ENVIRONMENT PROGRAMME, 2019). Segundo a Ellen MacArthur Foundation (2019) cerca de 40\% dos resíduos sólidos urbanos são provenientes das atividades de construção e demolição (CD), além disso, o consumo de materiais devido ao aumento da urbanização passará, de forma estimada, de 40 bilhões de toneladas em 2010 para 90 bilhões de toneladas em 2050. De acordo com a International Energy Agency, em 2018, a construção civil foi responsável pelo consumo de cerca de 36\% do uso do energia final, além de uma porcentagem expressiva de 39\%, relacionada a emissão de dióxido de carbono (sendo que $11 \%$ desse valor refere-se a produção de materiais e produtos 
como cimento, vidro e aço) (UNITED NATIONS ENVIRONMENT PROGRAMME, 2019; ZANNI et al., 2018)

Por isso, a importância do trabalho em saber o que está sendo estudado sobre economia circular, design for deconstruction e pré-fabricados, além da integração entre os três, no âmbito da construção civil para reduzir os subprodutos sem valor agregados. Assim, este trabalho tem como objetivo principal fazer uma revisão da literatura de maneira exploratória, por meio de artigos de pesquisa e revisão de artigos na base de dados Science Direct de 2014 a 2020, realizado em quatro etapas para identificar a relação entre os conceitos Economia circular, Design for Deconstruction e uso de Pré-fabricados para redução de excedentes sem valor agregado na construção civil, denominados em muitos estudos de resíduos/desperdícios.

\section{Revisão da Literatura}

\subsection{Economia Circular}

Com objetivo de reduzir os desperdícios pela reestruturação, de maneira equilibrada, do uso da matéria-prima para o consumo e seu respectivo descarte, a economia circular (EC) baseia-se no método dos 3R (redução, reutilização e reciclagem). Ao contrário da economia linear, a EC tende a usar os produtos considerados sem valor agregado como insumos para realimentar o ciclo de vida da construção, ou seja, apresenta-se como um fluxo em um ciclo fechado (BAO; LU, 2020; HUANG et al., 2018; LÓPEZ et al., 2020).

O estudo feito por Ghaffar et al., (2020), com stakeholders do setor de Resíduos de Construção e Demolição (CDW) do Reino Unido constatou que o método dos 3R, aderido pela CE, apresenta como principais gargalos para reciclagem/reutilização: (i) a logística ; (ii) o custo; e (iii) a regulação do tempo. Outros autores ainda acrescentam problemas como: (i) a falta de transparência e eficiência das políticas da CD; (ii) designs inapropriados ou pouco otimizados; (ii) as mudanças de última hora no projeto; (iii) a falta de planejamento e controle da qualidade; (iv) o desperdício de matéria-prima; (v) a baixa adesão de pré-fabricados; (vi) as investigações detalhadas das propriedades do material (principalmente relacionadas às capacidades estruturais, que necessitam de testes de desempenho, podendo aumentar o custo do produto); (vii) o elevado custo em relação à triagem dos materiais e reprocessamento; (viii) e a falta de 
incentivo do mercado (AJAYI et al., 2017; HUANG et al., 2018; MAHPOUR, 2018; MUNARO et al., 2020).

Por outro lado, a aderência da EC na indústria da construção civil pode ser uma solução para uma importante lacuna que envolve questões ambientais como a emissão de gases de efeito estufa, o consumo de energia e recursos naturais, a poluição, a degradação do solo e o aumento de aterros sanitários que, de acordo com Aslam et al., (2020) ocorre devido ao crescimento da urbanização e de sua respectiva geração de resíduos tanto de construção como de demolição. Assim, as práticas EC, deveriam ser empregadas desde a concepção do projeto até sua execução, englobando a gestão dos resíduos e, até mesmo, o design (BAO; LU, 2020; JIN et al., 2019; LÓPEZ et al., 2020).

\subsection{Design}

De acordo com López et al., (2020), o design de projetos para construção necessita de estratégias qualificadas que atendam a EC e apresenta três métodos principais que devem ser adotados: (i) o projeto para prevenção de resíduos; (ii) o projeto para desmontagem e desconstrução (DfD); e (iii) a utilização de elementos pré-fabricados.

O conceito de DfD propicia um movimento cíclico durante as fases da construção, mediante a reciclagem de materiais, a reutilização de elementos e, até mesmo, o reaproveitamento total da construção com conexões entre os elementos, englobando assim o projeto para prevenção de resíduos (JAILLON; POON, 2014). Apesar da barreira cultural pautada na preferência por construções dispendiosas, que visam uma vida útil maior, o DfD apresenta-se como uma nova perspectiva às mudanças futuras e que podem ser incorporadas na construção (AKANBI et al., 2019). Assim, para aumentar a eficiência do DfD, principalmente quanto à parte da desmontagem, torna-se necessária a sincronia de tecnologias como o BIM (Building Information Modeling), um software capaz de armazenar todos os detalhes da construção (IACOVIDOU et al., 2018; JIN et al., 2019; LI et al., 2020).

\subsection{Pré-fabricados}

Akinade et al., (2017) afirmam que a aplicabilidade de componente pré-fabricados em substituição aos processos tradicionais de construção, assegura uma padronização, evitando modificações improvisadas nos espaços construtivos. Um estudo feito por Hao et al., (2020), 
na China, aponta que a utilização da pré-fabricação no ambiente da construção civil pode reduzir em aproximadamente $15 \%$ as emissões de carbono durante o estágio de materialização.

\section{Metodologia}

Este trabalho caracteriza-se como uma revisão bibliográfica de caráter exploratório, que tem como objetivo pesquisar trabalhos publicados sobre a temática dos subprodutos sem valor agregado gerados na construção civil, relacionados com os conceitos de economia circular, design for deconstruction e o uso de pré-fabricados. A busca foi realizada na base de dados Science Direct incluindo artigos nacionais e internacionais dos últimos 7 anos.

Entende-se por pesquisa bibliográfica um método para levantamento de materiais com uma cobertura ampla do assunto a ser pesquisado. A pesquisa exploratória garante diversos ângulos sobre o estudo, além de possuir planejamento flexível e dessa forma torna-se uma ferramenta para o desenvolvimento da revisão bibliográfica (GIL, 2008).

A pesquisa foi dividida em quatro etapas:

a) Etapa 1: busca realizada a partir da base de dados Science Direct combinadas com operador booleano AND. Desta forma foram pesquisados: "Circular Economy” (EC) and "Construction and demolition waste" (CDW) and "Design for deconstruction" (DfD) and "Prefabricated" (PF), sem obter resultados;

b) Etapa 2: Foi realizada uma nova pesquisa no mesmo banco de dados, porém combinando as palavras em pares e filtrando artigos de revisão e artigos de pesquisas de 2014 a 2020. Desta forma foram encontrados 71 resultados de acordo com agrupamento dois a dois. Desses, apenas, os que apresentavam relação direta com a construção e métodos para minimizar os resíduos foram selecionados, excluindo os artigos duplicados e os demais que não apresentavam as características de inclusão. Finalmente, foram obtidos e analisados integralmente 18 artigos;

c) Etapa 3: Combinou-se os conceitos "Circular Economy" (EC), "Design for deconstruction" (DfD) e o método "Prefabricated" (PF) com as palavras "Waste" (W) e "Construction" (C) conectados com operador booleano AND, para uma nova busca no banco de dados da Science Direct. Desta vez, foram filtrados apenas artigos de revisão e artigos de pesquisas entre os anos de 2014 a 2020;

d) Etapa 4: Por fim, os resultados foram analisados por meio do software VosViewer (uma ferramenta para elaboração de redes bibliométricas), a fim de analisar as palavras-chaves 
mais utilizadas nos títulos e resumos das buscas, com base na rede de ocorrência que agrupa os termos em cluster, que relaciona as palavras de acordo com a área (VAN ECK; WALTMAN, 2013).

\section{Resultados}

Diante das buscas realizadas no banco de dados Science Direct constatamos uma variação significativa de resultados, a depender da associação das palavras-chaves. A palavra chave "Circular Economy" apresentou predominância na pesquisa de acordo com a Tabela 1 dos primeiros resultados das buscas.

Tabela 1 - Combinação de palavras-chave

\begin{tabular}{lcccc}
\hline \multicolumn{1}{c}{ Combinação } & $\begin{array}{c}\text { Artigos de } \\
\text { revisão }\end{array}$ & Percentual & $\begin{array}{c}\text { Artigos de } \\
\text { pesquisa }\end{array}$ & Percentual \\
\hline$(\mathrm{EC})$ and (CDW) & 07 & $9,86 \%$ & 26 & $36,62 \%$ \\
$(\mathrm{EC})$ and (DfD) & 00 & $0,0 \%$ & 01 & $1,41 \%$ \\
$(\mathrm{EC})$ and (PF) & 01 & $1,41 \%$ & 03 & $4,22 \%$ \\
$(\mathrm{CDW})$ and (DfD) & 02 & $2,82 \%$ & 08 & $11,27 \%$ \\
$(\mathrm{CDW})$ and (PF) & 02 & $2,82 \%$ & 05 & $7,04 \%$ \\
$(\mathrm{DfD})$ and (PF) & 03 & $4,22 \%$ & 13 & $18,31 \%$ \\
\hline \multicolumn{5}{c}{ Fonte: próprios autores }
\end{tabular}

Fonte: próprios autores

De acordo com os resultados apresentados na Tabela 1 fica evidente que a associação da "Circular Economy" com a "Construction and demolition waste" resultaram em números superiores de artigos de revisão e de pesquisa, em relação às outras combinações, confirmando assim as preferências dos pesquisadores em relação ao tema. Dentre os 71 artigos encontrados foram filtrados e aplicados os critérios de exclusão significantes ao estudo resultando em 18 artigos.

Mediante uma análise temporal, quantitativa dos estudos e suas respectivas origens, nota-se que tanto na Europa quanto na China, as pesquisas para redução de resíduos apresentam maior índice, além disso, as publicações sobre EC e métodos para redução e gestão de resíduos aumentaram nos últimos 3 anos revelando que assunto apresenta aumento sobre a relevância exploratória (MUNARO et al., 2020). Além disso, a adoção dos conceitos do Design for Deconstruction e o método de pré-fabricados podem ser englobados no EC para torná-lo mais eficazes. Outra constatação, consiste em um maior número de publicações, com datas mais recentes, no periódico Journal of Cleaner Production conforme o Quadro 1. 


\begin{tabular}{|c|c|c|c|c|c|}
\hline Título & Autor(es) & Periódico & $\begin{array}{l}\text { Ano de } \\
\text { pubbli- } \\
\text { cação }\end{array}$ & $\begin{array}{l}\text { Índices } \\
\text { de } \\
\text { citação }\end{array}$ & Palavras-chaves \\
\hline $\begin{array}{l}\text { Pathways to circular } \\
\text { construction: } \\
\text { integrated } \\
\text { management } \\
\text { construction of } \\
\text { demolition waste for } \\
\text { resource recovery }\end{array}$ & $\begin{array}{l}\text { Seyed, H. G.; } \\
\text { Matthew, B.; } \\
\text { Nuhu, B. }\end{array}$ & $\begin{array}{l}\text { Journal of } \\
\text { Cleaner } \\
\text { Production }\end{array}$ & 2020 & 14 & $\begin{array}{c}\text { Circular } \\
\text { construction; } \\
\text { Construction and } \\
\text { demolition waste; } \\
\text { Re-use; Recycle; } \\
\text { Resource recovery }\end{array}$ \\
\hline $\begin{array}{l}\text { The circular economy } \\
\text { in the construction and } \\
\text { demolition waste } \\
\text { sector - A review and } \\
\text { an integrative model } \\
\text { approach. }\end{array}$ & $\begin{array}{l}\text { Luis, A. L. R.; } \\
\text { Xavier, R. R.; } \\
\text { Santiago, G. D. }\end{array}$ & $\begin{array}{l}\text { Journal of } \\
\text { Cleaner } \\
\text { Production }\end{array}$ & 2020 & 8 & $\begin{array}{l}\text { Construction and } \\
\text { demolition waste; } \\
\text { Material recovery; } \\
\text { Waste management; } \\
\text { Circular economy }\end{array}$ \\
\hline $\begin{array}{l}\text { Towards circular and } \\
\text { more sustainable } \\
\text { buildings: A } \\
\text { systematic literature } \\
\text { review on the circular } \\
\text { economy in the built } \\
\text { environment }\end{array}$ & $\begin{array}{l}\text { Mayara, R. M.; } \\
\text { Sergio, F. T.; } \\
\text { Luís, B. }\end{array}$ & $\begin{array}{l}\text { Journal of } \\
\text { Cleaner } \\
\text { Production }\end{array}$ & 2020 & 2 & $\begin{array}{c}\text { Circular economy; } \\
\text { Construction; } \\
\text { Built environment; } \\
\text { Systematic literature } \\
\text { review. }\end{array}$ \\
\hline $\begin{array}{l}\text { Research trend of the } \\
\text { application } \\
\text { information } \\
\text { technologies in } \\
\text { construction and } \\
\text { demolition waste } \\
\text { management }\end{array}$ & $\begin{array}{c}\text { Clyde, Z. L.; } \\
\text { Yiyu, Z.; Bing, } \\
\text { X.; Bo, Y.; } \\
\text { Vivian, W.Y.T.; } \\
\text { Zhe, C.; Yingyi, } \\
\text { Y. }\end{array}$ & $\begin{array}{l}\text { Journal of } \\
\text { Cleaner } \\
\text { Production }\end{array}$ & 2020 & 1 & $\begin{array}{l}\text { Construction and } \\
\text { demolition waste; } \\
\text { Information } \\
\text { management; } \\
\text { Literature review }\end{array}$ \\
\hline $\begin{array}{l}\text { Disassembly and } \\
\text { deconstruction } \\
\text { analytics system (D- } \\
\text { DAS) for construction } \\
\text { in a circular economy }\end{array}$ & $\begin{array}{c}\text { Lukman, A. A; } \\
\text { Lukumon, O. } \\
\text { O.; Kamil, O.; } \\
\text { Muhammad B.; } \\
\text { Olugbenga O. } \\
\text { A.; } \\
\text { Anuoluwapo, O. } \\
\text { A.; Juan, M. D. } \\
\text { D.; Hakeem, A. } \\
\text { O. }\end{array}$ & $\begin{array}{l}\text { Journal of } \\
\text { Cleaner } \\
\text { Production }\end{array}$ & 2019 & 12 & $\begin{array}{c}\text { Disassembly and } \\
\text { deconstruction } \\
\text { analytics (D-DAS); } \\
\text { Building information } \\
\text { modelling (BIM); } \\
\text { End-of-Life; } \\
\text { Circular economy; } \\
\text { Design for } \\
\text { deconstruction; } \\
\text { Design for } \\
\text { disassembly }\end{array}$ \\
\hline $\begin{array}{l}\text { Evaluating the } \\
\text { transition towards } \\
\text { cleaner production in } \\
\text { the construction and } \\
\text { demolition sector of } \\
\text { China: A review }\end{array}$ & $\begin{array}{l}\text { Patrizia, G.; Xi } \\
\text { J; Gengyuan, L.; } \\
\text { Sergio U. }\end{array}$ & $\begin{array}{l}\text { Journal of } \\
\text { Cleaner } \\
\text { Production }\end{array}$ & 2018 & 37 & $\begin{array}{c}\text { Construction and } \\
\text { demolition wastes; } \\
\text { Cleaner production; } \\
\text { Circular economy; } \\
\text { Sustainable } \\
\text { construction }\end{array}$ \\
\hline Título & Autor(es) & Periódico & $\begin{array}{l}\text { Ano de } \\
\text { pubbli- } \\
\text { cação }\end{array}$ & $\begin{array}{l}\text { Índices } \\
\text { de } \\
\text { citação }\end{array}$ & Palavras-chaves \\
\hline
\end{tabular}




\begin{tabular}{|c|c|c|c|c|c|}
\hline $\begin{array}{l}\text { Science mapping } \\
\text { approach to assisting } \\
\text { the review of } \\
\text { construction and } \\
\text { demolition waste } \\
\text { management research } \\
\text { published between } \\
2009 \text { and } 2018\end{array}$ & $\begin{array}{l}\text { Ruoyu, J.; } \\
\text { Hongping, Y.; } \\
\text { Qian, C. }\end{array}$ & $\begin{array}{l}\text { Resources, } \\
\text { Conservatio } \\
\mathrm{n} \text { and } \\
\text { Recycling }\end{array}$ & 2019 & 47 & $\begin{array}{c}\text { Construction and } \\
\text { demolition waste; } \\
\text { Sustainable } \\
\text { development; } \\
\text { Science mapping; } \\
\text { Waste management; } \\
\text { Scientometric } \\
\text { analysis; Literature } \\
\text { review }\end{array}$ \\
\hline $\begin{array}{l}\text { Construction and } \\
\text { demolition waste best } \\
\text { management practice } \\
\text { in Europe }\end{array}$ & $\begin{array}{l}\text { Gálvez-Martos, } \\
\text { J. L.; Styles, D.; } \\
\text { Schoenberger, } \\
\text { H.; Zeschmar- } \\
\text { Lahl, B. }\end{array}$ & $\begin{array}{l}\text { Resources, } \\
\text { Conservatio } \\
\mathrm{n} \text { and } \\
\text { Recycling }\end{array}$ & 2018 & 108 & $\begin{array}{c}\text { Construction and } \\
\text { demolition waste; } \\
\text { Circular economy; } \\
\text { Recycling; Re-use; } \\
\text { Best practices; } \\
\text { Environmental } \\
\text { management; Waste } \\
\text { management; Waste } \\
\text { logistics; Recycled } \\
\text { aggregates; } \\
\text { Plasterboard }\end{array}$ \\
\hline $\begin{array}{l}\text { Construction and } \\
\text { demolition waste } \\
\text { management in China } \\
\text { through the } 3 \mathrm{R} \\
\text { principle }\end{array}$ & $\begin{array}{l}\text { Huang, B.; } \\
\text { Wang, X.; Kua, } \\
\text { H.; Geng, Y.; } \\
\text { Bleischwit, R.; } \\
\text { Ren, J. }\end{array}$ & $\begin{array}{l}\text { Resources, } \\
\text { Conservatio } \\
\text { n and } \\
\text { Recycling }\end{array}$ & 2018 & 108 & $\begin{array}{l}\text { Construction and } \\
\text { demolition waste; } \\
\text { China; Management; } \\
\text { Circular economy }\end{array}$ \\
\hline $\begin{array}{lr}\text { Prioritizing } & \text { barriers to } \\
\text { adopt } & \text { circular } \\
\text { economy } & \text { in } \\
\text { construction } & \text { and } \\
\text { demolition } & \text { waste } \\
\text { management } & \end{array}$ & Amirreza, M. & $\begin{array}{l}\text { Resources, } \\
\text { Conservatio } \\
\mathrm{n} \text { and } \\
\text { Recycling }\end{array}$ & 2018 & 41 & $\begin{array}{l}\text { Potential barrier; } \\
\text { Circular economy; } \\
\text { C\&D waste } \\
\text { management; } \\
\text { Prioritization; Fuzzy } \\
\text { TOPSIS }\end{array}$ \\
\hline $\begin{array}{l}\text { Waste minimisation } \\
\text { through } \\
\text { deconstruction: A BIM } \\
\text { based } \\
\text { Deconstructability } \\
\text { Assessment Score } \\
\text { (BIM-DAS) }\end{array}$ & $\begin{array}{c}\text { Olugbenga, O. } \\
\text { A; Lukumon O. } \\
\text { O;Muhamma,B. } \\
\text {; Saheed, O. } \\
\text { A.;Hakeem, A. } \\
\text { O.; Hafiz, A. } \\
\text { A.;Sururah, A. } \\
\text { R. B. }\end{array}$ & $\begin{array}{c}\text { Resources, } \\
\text { Conservatio } \\
\mathrm{n} \text { and } \\
\text { Recycling }\end{array}$ & 2015 & 73 & $\begin{array}{l}\text { Building information } \\
\text { modelling; Building } \\
\text { deconstruction; } \\
\text { Design for } \\
\text { deconstruction; } \\
\text { Demolition waste } \\
\text { minimization; } \\
\text { Design performance } \\
\text { assessment; Scoring } \\
\text { scheme }\end{array}$ \\
\hline $\begin{array}{lr}\text { Developing efficient } \\
\text { circularity } \\
\text { construction for } \\
\text { demolition and } \\
\text { management in fast } \\
\text { emerging economies: } \\
\text { Lessons learned from } \\
\text { Shenzhen, China }\end{array}$ & $\begin{array}{l}\text { Zhikang, B; } \\
\text { Weisheng L. }\end{array}$ & $\begin{array}{l}\text { Science of } \\
\text { The Total } \\
\text { Environment }\end{array}$ & 2020 & 5 & $\begin{array}{c}\text { Construction and } \\
\text { demolition waste; } \\
\text { Waste management; } \\
\text { Circular economy; } \\
\text { Emerging } \\
\text { economies; } \\
\text { Shenzhen; China }\end{array}$ \\
\hline Título & Autor(es) & Periódico & $\begin{array}{l}\text { Ano de } \\
\text { publi- } \\
\text { cação }\end{array}$ & $\begin{array}{l}\text { Índices } \\
\text { de } \\
\text { citação }\end{array}$ & Palavras-chaves \\
\hline
\end{tabular}




\begin{tabular}{|c|c|c|c|c|c|}
\hline $\begin{array}{l}\text { Carbon emission } \\
\text { reduction in } \\
\text { prefabrication } \\
\text { construction during } \\
\text { materialization stage: } \\
\text { A BIM-based life- } \\
\text { cycle assessment } \\
\text { approach }\end{array}$ & $\begin{array}{l}\text { Hao, J. L.; } \\
\text { Cheng, B.; Lu, } \\
\text { W.; Xu, J.; } \\
\text { Wang, J.; Bu, } \\
\text { W.; Guo, Z. }\end{array}$ & $\begin{array}{l}\text { Science of } \\
\text { The Total } \\
\text { Environment }\end{array}$ & 2020 & 3 & $\begin{array}{c}\text { Carbon emissions; } \\
\text { Prefabrication; } \\
\text { Materialization } \\
\text { stage; } \\
\text { BIM; } \\
\text { Life cycle } \\
\text { assessment }\end{array}$ \\
\hline $\begin{array}{l}\text { Review } \\
\text { construction } \\
\text { demolition and } \\
\text { management in } \\
\text { and USA } \\
\text { and } \\
\end{array}$ & $\begin{array}{c}\text { Aslam, M.S.; } \\
\text { Huang, B.; Cui } \\
\text { L. }\end{array}$ & $\begin{array}{c}\text { Journal of } \\
\text { Environment } \\
\text { al } \\
\text { Management }\end{array}$ & 2020 & 2 & $\begin{array}{l}\text { Construction and } \\
\text { demolition waste } \\
\text { management; } \\
\text { Urbanization; } \\
\text { Circular economy; } \\
\text { Policies }\end{array}$ \\
\hline $\begin{array}{l}\text { The use of smart } \\
\text { technologies in } \\
\text { enabling construction } \\
\text { components reuse: A } \\
\text { viable method or a } \\
\text { problem creating } \\
\text { solution? }\end{array}$ & $\begin{array}{l}\text { Iacovidou, E.; } \\
\text { Purnell, P.; Lim, } \\
\text { M. K. }\end{array}$ & $\begin{array}{c}\text { Journal of } \\
\text { Environment } \\
\text { al } \\
\text { Management }\end{array}$ & 2018 & 19 & $\begin{array}{c}\text { Construction and } \\
\text { demolition waste } \\
\text { (CDW); Structural } \\
\text { components; RFID; } \\
\text { Reuse; Information } \\
\text { flow; Lifecycle } \\
\text { valuation }\end{array}$ \\
\hline $\begin{array}{l}\text { Attributes of design for } \\
\text { construction waste } \\
\text { minimization: A case } \\
\text { study of waste-to- } \\
\text { energy project }\end{array}$ & $\begin{array}{c}\text { Ajayi, S. O.; } \\
\text { Oyedele, L. O.; } \\
\text { Bilal, M.; } \\
\text { Akinade, O. O.; } \\
\text { Alaka, H. A.; } \\
\text { Owolabi, H. A.; } \\
\text { Kadiri, K. O. }\end{array}$ & $\begin{array}{c}\text { Renewable } \\
\text { and } \\
\text { Sustainable } \\
\text { Energy } \\
\text { Reviews }\end{array}$ & 2017 & 22 & $\begin{array}{l}\text { Design out waste; } \\
\text { Design documents; } \\
\text { Deconstruction; } \\
\text { Buildability; } \\
\text { Construction waste; } \\
\text { Waste-to-Energy }\end{array}$ \\
\hline $\begin{array}{lr}\text { Design for } & \text { feconstruction (DfD): } \\
\text { Deritical success factors } \\
\text { Criverting end-of- } \\
\text { for diver } \\
\text { life waste from } \\
\text { landfills }\end{array}$ & $\begin{array}{c}\text { Akinade, O.O.; } \\
\text { Oyedele, L. O.; } \\
\text { Ajayi, S. O.; } \\
\text { Bilal, M.; } \\
\text { Alaka, H. A.; } \\
\text { Owolabi, H. A.; } \\
\text { Bello, S. A.; } \\
\text { Jaiyeoba, B. E.; } \\
\text { Kadiri, K. }\end{array}$ & $\begin{array}{c}\text { Waste } \\
\text { Management }\end{array}$ & 2017 & 41 & $\begin{array}{c}\text { Building } \\
\text { deconstruction; } \\
\text { Design for } \\
\text { deconstruction; End- } \\
\text { of-life material } \\
\text { recovery; } \\
\text { Sustainable } \\
\text { construction; } \\
\text { Material reuse; } \\
\text { Critical success } \\
\text { factors } \\
\end{array}$ \\
\hline $\begin{array}{l}\text { Life cycle design and } \\
\text { prefabrication in } \\
\text { buildings: A review } \\
\text { and case studies in } \\
\text { Hong Kong }\end{array}$ & $\begin{array}{l}\text { Jaillon, L.; } \\
\text { Poon, C. S. }\end{array}$ & $\begin{array}{l}\text { Automation } \\
\text { in } \\
\text { Construction }\end{array}$ & 2014 & 91 & $\begin{array}{l}\text { Design for } \\
\text { deconstruction; } \\
\text { High-rise buildings; } \\
\text { Hong Kong; Life } \\
\text { cycle design; } \\
\text { Prefabrication }\end{array}$ \\
\hline
\end{tabular}

Fonte: próprios autores

A terceira etapa, também corroborou o fato de que o termo "Circular Economy" apresenta-se como foco nas pesquisas, categorizando $74,2 \%$ dos resultados, como mostra a Tabela 2. 
Tabela 2 - Combinação de palavras-chave

\begin{tabular}{lcccc}
\hline Combinação & $\begin{array}{c}\text { Artigos de } \\
\text { revisão }\end{array}$ & Percentual & $\begin{array}{c}\text { Artigos de } \\
\text { pesquisa }\end{array}$ & Percentual \\
\hline (EC) and (W) and (C) & 26 & $13,68 \%$ & 115 & $60,53 \%$ \\
$(\mathrm{DfD})$ and (W) and (C) & 00 & $0,0 \%$ & 07 & $3,69 \%$ \\
$(\mathrm{PF})$ and (W) and (C) & 05 & $2,63 \%$ & 37 & $19,47 \%$ \\
\hline
\end{tabular}

Fonte: próprios autores

Com base nos artigos encontrados na terceira etapa, foram montadas redes bibliométricas no VosViewer, para analisar as relações entre as palavras-chaves (VAN ECK; WALTMAN, 2013). A primeira combinação "Circular Economy" (EC), "Waste" (W) e "Construction" (C) resultou em 527 palavras-chaves e após serem agrupadas formou-se três clusters, separados por cores distintas, de acordo com a Figura 1. Os clusters foram agrupados da seguinte forma: o primeiro, conectando resíduos de construção e demolição (com 14 ocorrências), reciclagem (12) e gestão de resíduos (11); o segundo, relacionando economia circular (74) e sustentabilidade (10); e o terceiro, avaliação do ciclo de vida (10).

Figura 1 - Rede de combinação: “Circular Economy” (EC), “Waste” (W) e "Construction” (C)

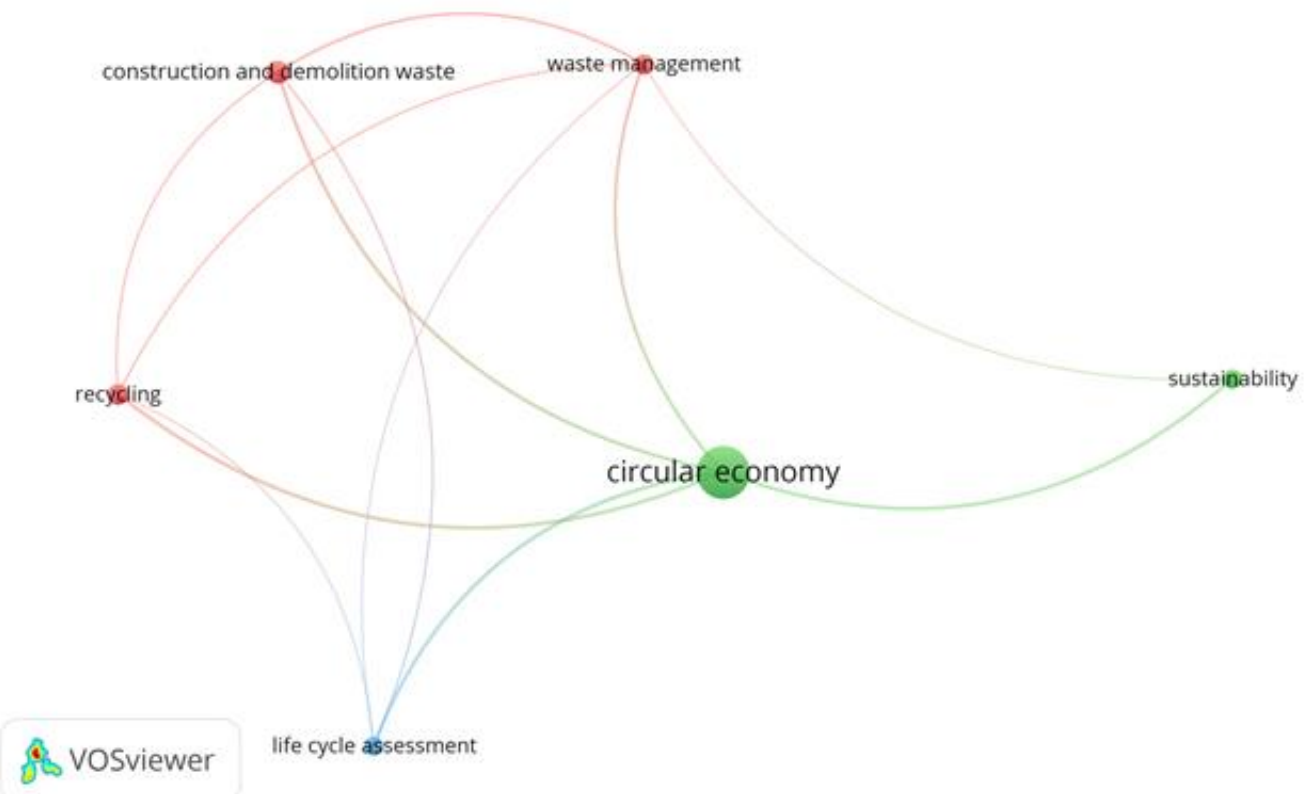

Fonte: Elaborado pelos autores com auxílio do Vosviewer

De acordo com a Figura 1 a interligação entre os clusters está evidenciada, principalmente em relação ao primeiro com a palavra-chave "Circular Economy" (EC).

A segunda combinação entre "Design for deconstruction" (DfD), "Waste” (W) e "Construction" (C) resultou em 36 palavras-chaves, o número mínimo de ocorrências foi determinado pelo próprio programa, pois a amostra era pequena, assim foram obtidos três clusters, separados por cores distintas, demostrado na Figura 2. O primeiro contendo: construção desconstrução (2), modelo de informação de construção (1), minimização de resíduos de demolição (1), avaliação de desempenho de projeto (1), esquema de pontuação (1); o segundo: projeto para desconstrução (3), edifícios de arranha-céus (1), Hong Kong (1), projeto do ciclo de vida(1), pré-fabricado (1); já o terceiro: fatores de sucesso críticos (1), recuperação material do fim da vida (1), reutilização material (1), construção utilizável (1). 
Figura 2 - Combinação entre "Design for deconstruction” (DfD), “Waste” (W) e "Construction” (C)

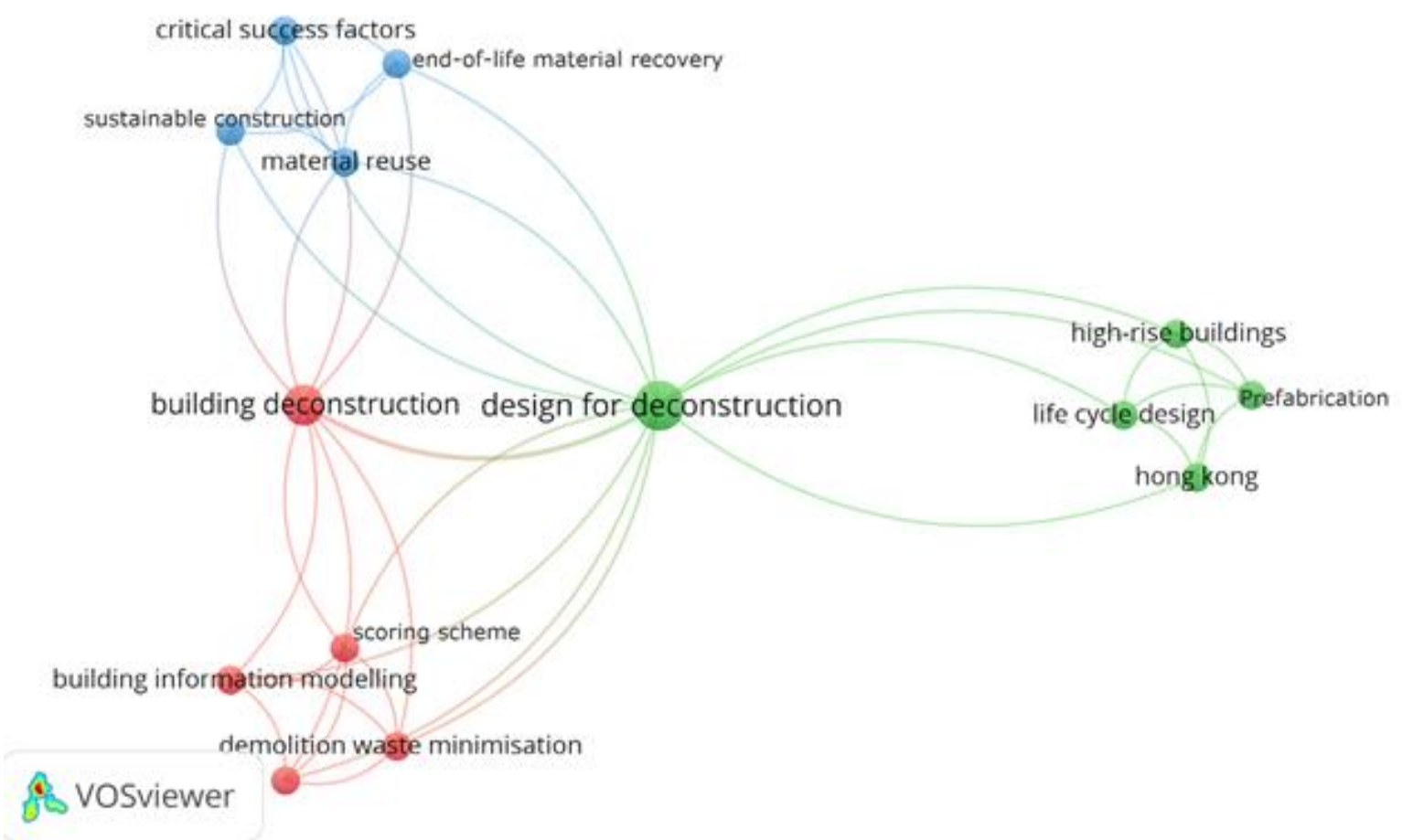

Fonte: Elaborado pelos autores com auxílio do Vosviewer

A Figura 2 apresenta os clusters melhor subdivido, relacionando no segundo cluster os conceitos de projeto para desconstrução com o uso de pré-fabricado.

A terceira e última combinação foi entre as palavras "Prefabricated" (PF), "Waste" (W) e "Construction" (C) resultando em 188 palavras-chaves que, após serem agrupadas, formou quatro clusters, conforme a Figura 3. Aqui, também, os clusters foram separados por cores distintas sendo: no primeiro as palavras BIM (com 2 ocorrências), avaliação do ciclo de vida (3), construção fora do local (2); no segundo redução de carbono (2), pré-fabricados (7) construção residencial (2); no terceiro resíduos de construção e demolição (4), desconstrução (2), edifício alto ( 2); no quarto design para desconstrução (2) e Hong Kong (2).

Figura 3 - Combinação entre as palavras "Prefabricated” (PF), “Waste” (W) e “Construction” (C) 


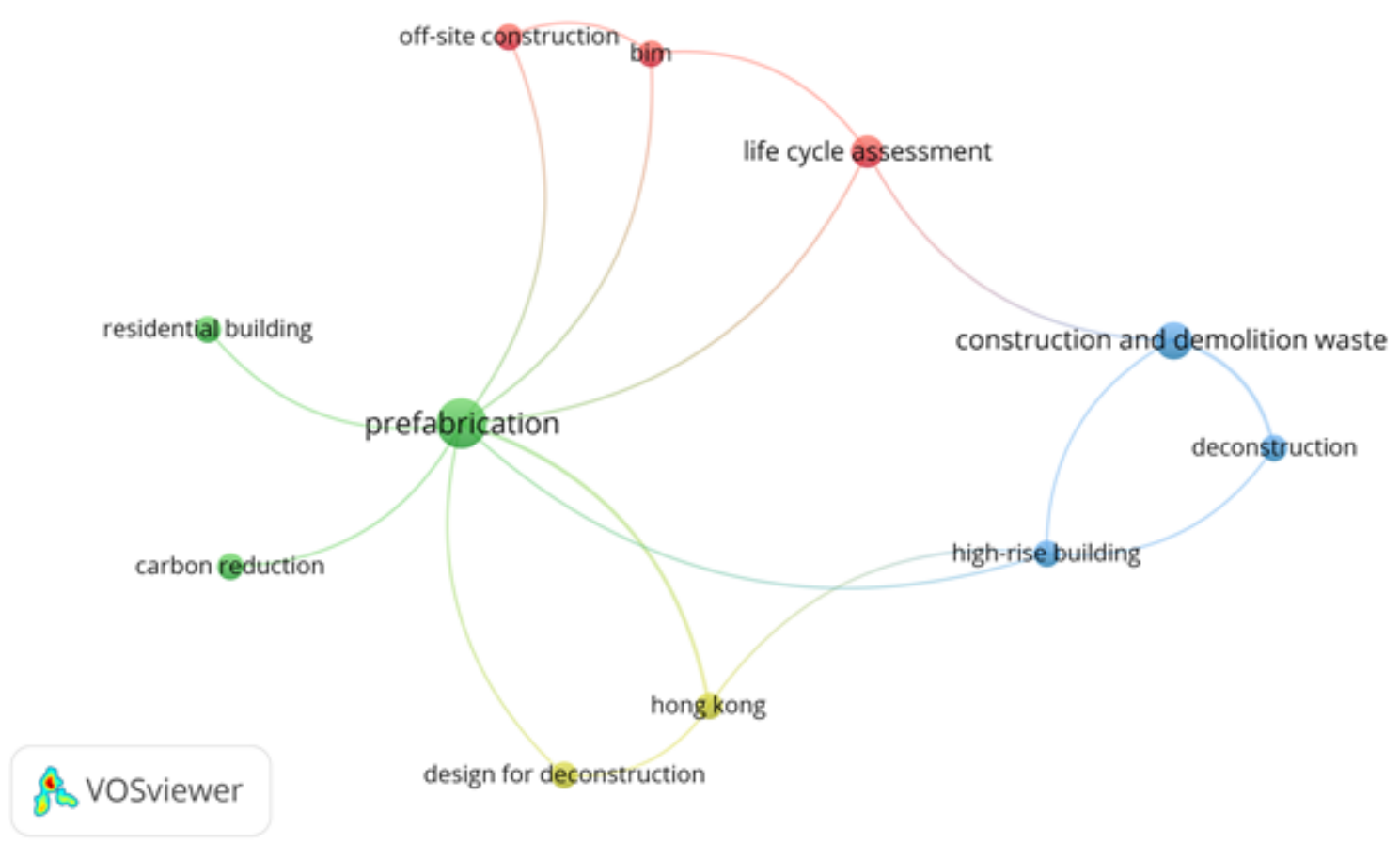

Fonte: Elaborado pelos autores com auxílio do Vosviewer

Já a Figura 3 o conceito de desconstrução aparece em dois clusters distintos, confirmando a tendência de ligação entre o uso de pré-fabricados com o conceito DfD. Além de relacionar CDW com avaliação do ciclo de vida e desconstrução.

\section{Conclusão}

A análise feita a partir dos periódicos selecionados na base de dados Science Direct, mostrou que a palavra-chave "Circular Economy", conforme todas as combinações realizadas nas buscas, apresentou-se com maior ocorrência. Entretanto, sua associação com o conceito Design for deconstruction e o método para uso de pré-fabricados mostrou-se ínfima, quando comparada, por exemplo, com os termos gestão de resíduos e resíduos de construção de demolição. Porém, por meio da análise das palavras-chaves com o software Vosviewer a relação entre Design for deconstruction e o método para uso de pré-fabricados excluindo-se o termo economia circular apresenta-se com mais frequência. Pode-se notar na Figura 2 que essa combinação se apresenta, inclusive, até nos mesmos clusters, além disso com a busca da palavras-chave "Prefabricated", "Waste" e "Construction" a desconstrução apareceu em dois clusters conforme ilustrado na Figura 3.

Além disso, foi possível verificar com os 18 artigos selecionados que, como era esperado, os artigos mais citados não são os mais recentes e o periódico Journal of Cleaner Production 
apresenta mais artigos na área investigada. Ademais a China, assim como a Europa, foi apontada em vários estudos de gestão de resíduos com economia circular.

Assim, conclui-se com a pesquisa que existe uma ascensão nos estudos com relação ao tema, além disso tanto o Design for deconstruction como o método para uso de pré-fabricados são ferramentas que apresentam bastante potencial para que os princípios do EC sejam cumpridos em função de diminuir os subprodutos sem valor agregado na construção civil, contudo faz-se necessário um maior aprofundamento nos estudos relacionando esses três campos.

\section{REFERÊNCIAS}

AJAYI, S. O.; OYEDELE, L. O.; AKINADE, O. O.; BILAL, M.; ALAKA, H. A.; OWOLABI, H. A.; KADIRI, K. O. Attributes of design for construction waste minimization: A case study of waste-to-energy project. Renewable and Sustainable Energy Reviews, v. 73, n. January, p. 1333-1341, 2017.

AKANBI, L. A.; OYEDELE, L. O.; OMOTESO, K.; BILAL, M.; AKINADE, O. O.; AJAYI, A. O.; DAVILA DELGADO, J. M.; OWOLABI, H. A. Disassembly and deconstruction analytics system (D-DAS) for construction in a circular economy. Journal of Cleaner Production, v. 223, p. 386-396, 2019.

AKINADE, O. O.; OYEDELE, L. O.; AJAYI, S. O.; BILAL, M.; ALAKA, H. A.; OWOLABI, H. A.; BELLO, S. A.; JAIYEOBA, B. E.; KADIRI, K. O. Design for Deconstruction (DfD): Critical success factors for diverting end-of-life waste from landfills. Waste Management, v. 60, p. 3-13, 2017.

ASLAM, M. S.; HUANG, B.; CUI, L. Review of construction and demolition waste management in China and USA. Journal of Environmental Management, v. 264, n. March, p. $110445,2020$.

BAO, Z.; LU, W. Developing efficient circularity for construction and demolition waste management in fast emerging economies: Lessons learned from Shenzhen, China. Science of the Total Environment, v. 724, p. 138264, 2020.

ELLEN MACARTHUR, F. Completando a figura: Como a economia circular ajuda a enfrentar as mudanças climáticas. p. 1-62, 2019. Disponível

em:<https://www.ellenmacarthurfoundation.org/publications $>$. Acesso 10 set. 2020.

GHAFFAR, S. H.; BURMAN, M.; BRAIMAH, N. Pathways to circular construction: An integrated management of construction and demolition waste for resource recovery. Journal of Cleaner Production, v. 244, p. 118710, 2020.

GIL, A. C. Delineamento da Pesquisa. 6. ed. São Paulo: Ed. ATLAS, 2008. v. 264

HAO, J. L.; CHENG, B.; LU, W.; XU, J.; WANG, J.; BU, W.; GUO, Z. Carbon emission reduction in prefabrication construction during materialization stage: A BIM-based life-cycle assessment approach. Science of the Total Environment, v. 723, p. 137870, 2020.

HUANG, B.; WANG, X.; KUA, H.; GENG, Y.; BLEISCHWITZ, R.; REN, J. Construction and demolition waste management in China through the $3 \mathrm{R}$ principle. Resources,

Conservation and Recycling, v. 129, n. April 2017, p. 36-44, 2018. 
IACOVIDOU, E.; PURNELL, P.; LIM, M. K. The use of smart technologies in enabling construction components reuse: A viable method or a problem creating solution? Journal of Environmental Management, v. 216, p. 214-223, 2018.

IEA. GlobalABC Roadmap for Buildings and Construction 2020-2050: Towards a zeroemission, efficient and resilient buildings and construction sector. Disponível em:< https://www.iea.org/reports/globalabc-roadmap-for-buildings-and-construction-2020-2050>. Acesso em: 10 jul. 2020.

JAILLON, L.; POON, C. S. Life cycle design and prefabrication in buildings: A review and case studies in Hong Kong. Automation in Construction, v. 39, p. 195-202, 2014.

JIN, R.; YUAN, H.; CHEN, Q. Science mapping approach to assisting the review of construction and demolition waste management research published between 2009 and 2018. Resources, Conservation and Recycling, v. 140, n. September 2018, p. 175-188, 2019.

LI, S.; FANG, Y.; WU, X. A systematic review of lean construction in Mainland China. Journal of Cleaner Production, v. 257, 2020.

LÓPEZ RUIZ, L. A.; ROCA RAMÓN, X.; GASSÓ DOMINGO, S. The circular economy in the construction and demolition waste sector $-\mathrm{A}$ review and an integrative model approach. Journal of Cleaner Production, v. 248, 2020.

MAHPOUR, A. Prioritizing barriers to adopt circular economy in construction and demolition waste management. Resources, Conservation and Recycling, v. 134, n. December 2017, p. 216-227, 2018.

MUNARO, M. R.; TAVARES, S. F.; BRAGANÇA, L. Towards circular and more sustainable buildings: A systematic literature review on the circular economy in the built environment. Journal of Cleaner Production, v. 260, 2020.

UNITED NATIONS ENVIRONMENT PROGRAMME. Towards a zero-emissions, efficient and resilient buildings and construction sector. 2019 Global Status report. [S. $l$.: s. n.]. E-book. Disponível em:< https://www.worldgbc.org/sites/default/files/UNEP 188_GABC_en (web).pdf>. Acesso 15 set. 2020.

VAN ECK, N. J.; WALTMAN, L. Manual. Leiden: Univeristeit Leiden, n. April, 2013. Disponível em: $\langle$ http://www.vosviewer.com/documentation/Manual_VOSviewer_1.6.1.pdf >. Acesso 20 set. 2020.

ZANNI, S.; SIMION, I. M.; GAVRILESCU, M.; BONOLI, A. Life Cycle Assessment Applied to Circular Designed Construction Materials. Procedia CIRP, v. 69, n. May 2018, p. 154-159, 2018. 